\title{
Using high density EEG to assess TMS treatment in patients with schizophrenia
}

Sara Marcu (1,2), Elena Pegolo (1,2), Eysteinn Ívarsson (3), Aron D. Jónasson (3), Viktor D. Jónasson (4), Romain Aubonnet (1), Paolo Gargiulo (1), Ovidiu C. Banea $(1,3)$

(1) Reykjavik University, School of Science and Engineering, Biomedical Engineering, Reykjavik, Iceland; (2) Department of Information Engineering, University of Padova, Italy; (3) Clinical Neurophysiology Unit, Neurology Department, National University Hospital of Iceland; (4) Psychiatric Department, National University Hospital of Iceland.

This article is distributed under the terms of the Creative Commons Attribution Noncommercial License (CC BY-NC 4.0) which permits any noncommercial use, distribution, and reproduction in any medium, provided the original author(s) and source are credited.

\begin{abstract}
We present preliminary results from the ongoing study entitled "Icelandic AVH-TMS" which aim is to study the effectiveness of repetitive transcranial magnetic stimulation (rTMS) treatment for patients with schizophrenia and with persistent auditory verbal hallucinations (AVH) using symptoms and psychometric scales and high-density EEG system (256 channels). The aim of the present work was to describe cortical topography of the auditory evoked responses like P50 and N100-P300 complex in healthy participants and patients with schizophrenia and to define a robust methodology of signal quantification using dense-array EEG. Preliminary data is shown for three healthy participants and three patients in baseline conditions and for two patients we show the results recorded before and after 10 days rTMS treatment. Our results show differences in sensory gating (P50 suppresion) and a stronger N100-P300 response to rare audio stimulus after the treatment. Moreover we show the value of assessing brain electrical activity from highdensity EEG (256 channels) analyzing the results in different regions of interest. However, it is premature and hazardous to assume that rTMS treatment effectiveness in patients with AVH can be assessed using P50 suppression ratio.
\end{abstract}

Key Words: Transcranial magnetic stimulation, schizophrenia, high density EEG, P50, P300.

Eur J Transl Myol 30 (1): 134-138, 2020

P50 suppression and P300 deficits in patients with schizophrenia were found to be of similar magnitude as findings reported in neuroimaging and neuropsychology. ${ }^{1}$ Dysfunction of sensory gating is of high interest in psychiatric research and has been studied mostly with P50, a positive amplitude wave occurring approximately $50 \mathrm{~ms}$ after an auditory stimulus, which likely reflects pre-attentive information processing. ${ }^{2}$ Sensory gating deficits using P50 suppression have been found in schizophrenia. ${ }^{3}$ P50 gating is not always related to cognitive deficits, ${ }^{4}$ and the effect of different treatments on P50 analyzed together with positive or negative symptoms were controversial showing that abnormal sensory gating is not directly associated with the expression of these symptoms. ${ }^{5}$ Event related potentials are the most elaborated ERPs in psychiatric studies. The components N100, P200, N200, P300 are elicited by the rare stimuli; whereas frequent stimuli elicit only N100 and P200. ${ }^{6}$ During a two-tone discrimination (oddball) task the mean amplitudes of the N100 and P300 auditory responses are decreased in patients with schizophrenia in comparison to the healthy participants. ${ }^{6,7}$

In 1999, Hoffman and colleagues started to explore the repetitive transcranial magnetic stimulation (rTMS) for the treatment of auditory verbal hallucinations (AVH). When the coil was directed at the left temporoparietal cortex, they were able to ameliorate pharmaco - resistant $\mathrm{AVH} .{ }^{8}$ Since then, many other studies and meta-analyses showed that rTMS is capable of reducing the frequency and severity of auditory hallucinations with significantly better symptom reduction for low-frequency rTMS as compared with placebo. ${ }^{9-11}$. According to the available literature, previous studies describing the effectiveness of rTMS as a treatment for patients with persistent AVH used mainly subjective measurements like psychometric scales. Within the "Icelandic AVH-TMS" study the goal is to assess rTMS treatment with symptoms scales and 
neurophysiological markers like ERPs, gamma and beta relative power or cortical silent period.

While the examination of P300 topography in chronically ill patients with schizophrenia and psychotic bipolar disorder was associated with a specific left-lateralized posterior abnormality, suggesting underlying posterior temporal lobe pathology with abnormalities of a generator located in the left superior temporal gyrus (STG), ${ }^{12,13}$ the topography of P50 remains largely unknown ${ }^{14}$. Where the P50 suppression and P300 waves show major changes or dysfunction at cortical level remain unclear as most studies reported $\mathrm{P} 300$ data at $\mathrm{Pz}$ and $\mathrm{Cz}$ electrodes while P50 researchers consistently reported analysis at $\mathrm{Cz}$ and thus only this location was used for analysis. ${ }^{1}$ The objective of this study was to present the method of P50 suppression and N100-P300 components measures from seven different brain locations (left anterior, left posterior, medial anterior, medial central, medial posterior, right anterior and right posterior) using high density EEG. Our hypotheses were that after rTMS treatment, P50 suppression ratio decreases (improved sensory gating) and N100-P300 voltage increases.

\section{Materials and Methods}

The patients have been recruited from the psychiatric wards and outpatient clinics through the National Hospital database of diagnosed schizophrenia patients, following the ICD-10 schizophrenia classification (F20). Only those still experiencing persistent auditory verbal hallucinations after finishing at least two 6-8 week drug prescription treatments have been selected. Permission from the Ethics Committee at the University Hospital of Iceland was obtained (approval no. 21.2018). Healthy participants were recruited by convenience sampling and went through Mini-International Neuropsychiatric Interview (M.I.N.I.).

\section{P50 suppression.}

The paired-click paradigm was performed to elicit the P50 component. A pure tone $(1500 \mathrm{~Hz}, 6-\mathrm{ms}$ duration at comfortable hearing noise) was used as the click sound and presented during a 500-ms interval through headphones. The interval between paired stimuli was 10 seconds. We presented 150 paired stimulus in 5 blocks with interstimulus interval of 10 seconds, which provided 25 minutes of EEG measurement. ${ }^{15}$ In consideration of participant load and ear comfort that could influence EEG measurement, we instructed participants to watch a silent film and presented auditory stimuli from headphones as mentioned above. The $S 1$ response was identified as the most prominent peak in the 40 - to 80 -ms post stimulus window (Figure 1). The preceding negative trough was used to calculate the $\mathrm{S} 1$ amplitude. For the $\mathrm{S} 2$ response, the positive peak with latency closest to that of the S1 peak was selected. P50 suppression was calculated as the ratio of the mean value of the $S 2$ amplitude to the mean value of the $\mathrm{S} 1$ amplitude (S2:S1). ${ }^{16,17}$

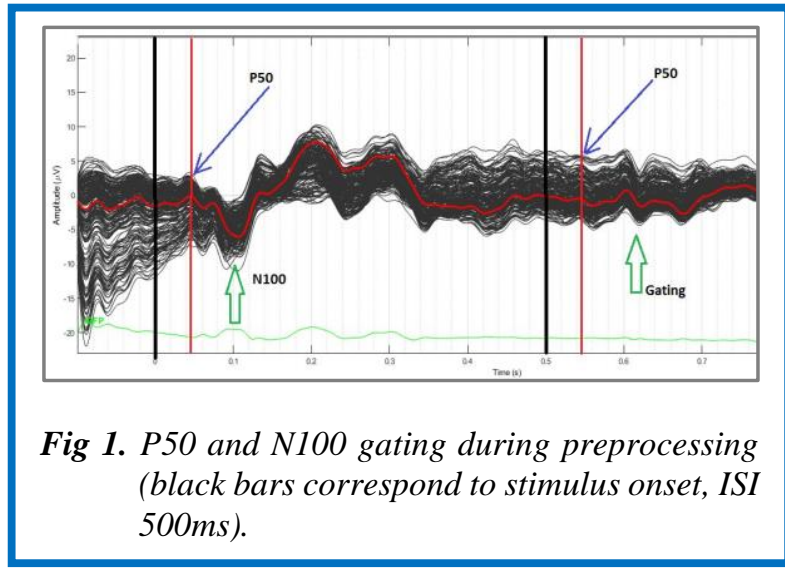

N100-P300 complex.

In our study P300 response was measured with an auditory oddball paradigm attention task. The recordings were carried out between 11:00 and 14:00 hours. The subjects were sitting in a comfortable chair with their eyes closed. The frequent $(\mathrm{F})$ and the rare $(\mathrm{R})$ auditory stimuli were presented binaurally through headphones at an interstimulus interval between tones of constant $1.1 \mathrm{sec}$. For each subject there was 1 trial of 160 tones which occurred randomly with a probability of $0.2 .{ }^{18} \mathrm{We}$ instructed the participants to pay attention at the rare stimuli without counting or moving a finger.

\section{EEG pre-processing and analysis.}

The EEG was recorded using 256 channel system (ANT Neuro, Netherlands) with an electrooculogram (EOG) electrode placed below the right eye, and a ground electrode placed on the left side of the neck. Data preprocessing and analysis was performed with Brainstorm ${ }^{19}$ and MATLAB 2018b. The data were sampled at $1024 \mathrm{~Hz}$ and re-referenced to the average of left and right mastoid electrodes (R19R, L19L). A bandpass filter was set between $0.1-80 \mathrm{~Hz},{ }^{20,21}$ and notch filter from $49-51 \mathrm{~Hz}$ was used to remove undesired monomorphic artifacts from $50 \mathrm{~Hz}$ mains electricity. ${ }^{22}$ Bad channels were removed when EEG voltage was greater than $\pm 80 \mu \mathrm{V}$; if more than $10 \%$ of the channels showed to much noise or bad signal, the whole trial was rejected. For P50 analysis the signals were digitized for an epoch of $500 \mathrm{~ms}$ starting $100 \mathrm{~ms}$ prior to the presentation of each auditory stimulus $(-100 \mathrm{~ms}$ to $+400 \mathrm{~ms})$ and for P300 response analysis the signals were digitized for each epoch of $1000 \mathrm{~ms}$ starting $100 \mathrm{~ms}$ prior to the presentation of each auditory stimulus $(-100$ to $+900 \mathrm{~ms}) .{ }^{20}$ Baseline correction was performed using pre stimulus $100 \mathrm{~ms}$ window and "bad" channels were removed and interpolated. Individual trials were visually inspected and rejected when indicative of excessive muscle activity, eye movements or other artifacts.

\section{The Regions of Interest}

The regions of interest (ROI) were defined using a matlab script, each of them being represented by 15 electrodes 


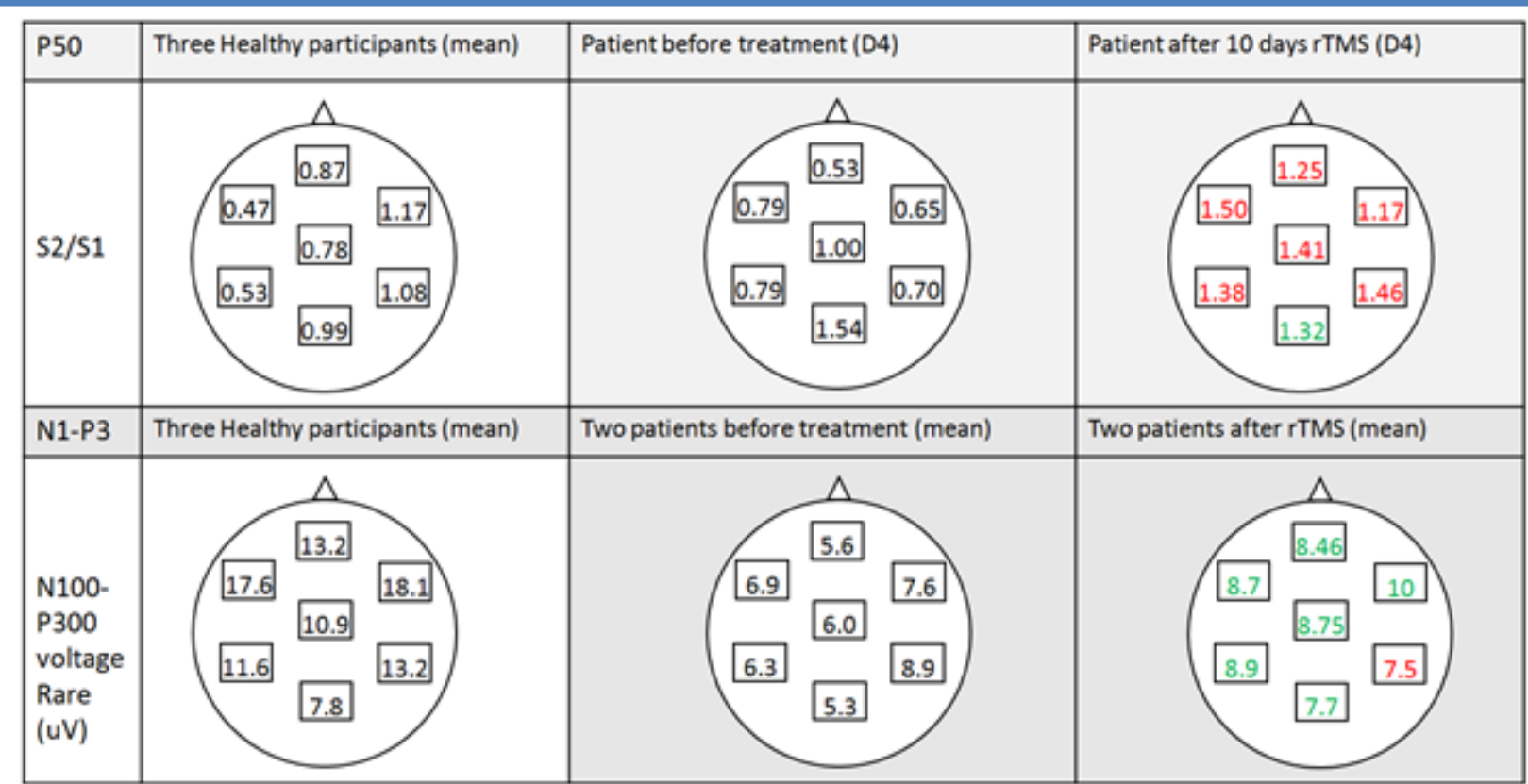

Fig 2. P50 suppression ratio in three healthy subjects and one patient with schizophrenia before and after rTMS treatment. N100-P300 voltage measurements in three healthy subjects and two patients with schizophrenia. Data was calculated $(u V)$ by averaging 15 channels responses corresponding to 7 ROI: left anterior, left posterior, medial anterior, medial central, medial posterior, right anterior and right posterior.

(105 electrodes out of 256) as follow: Left Anterior (LA), Left Posterior (LP), Medial Anterior (MA), Medial Central (MC), Medial Posterior (MP), Right Anterior (RA) and Right Posterior (RP). Fifteen electrodes were selected from 3 parallel lines for each region. We measured the peak-to-peak P50 amplitude from a preceding negative trough to the positive peak at 30-70 ms range from the stimulus onset. N100-P300 complex values for each ROI were calculated as the difference between the most negative voltage value and the most positive voltage value within the time range of 80-500 ms. In this work P50 suppression ratio and N1-P3 wave's signals were represented as the average of the fifteen channels of every ROI.

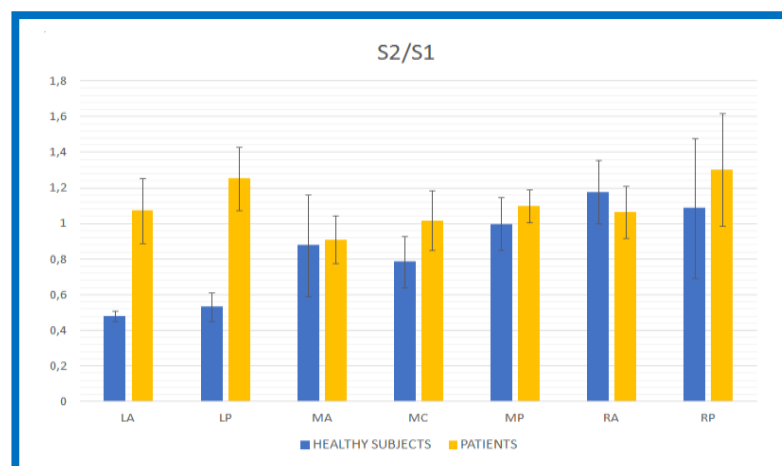

Fig 3. P50 gating in three healthy participants and three patients with schizophrenia (baseline).

\section{Results and Discussion}

Recording event related potentials with high density EEG system is challenging and difficult. Our preliminary data showed major P50 suppression (reduced ratio) in healthy participants than in the patients group (Figures 2 and 3). The patients showed higher ratios on the left anterior and left posterior regions suggesting that these regions might be functionally affected or that the gating in healthy participants is higher on the left anterior and left temporoparietal cortex (Figure 3). N100-P300 components were obtained and visible in the healthy group while patients with schizophrenia showed reduced or absent deviant stimulus responses before the treatment, which changed and was more visible after the

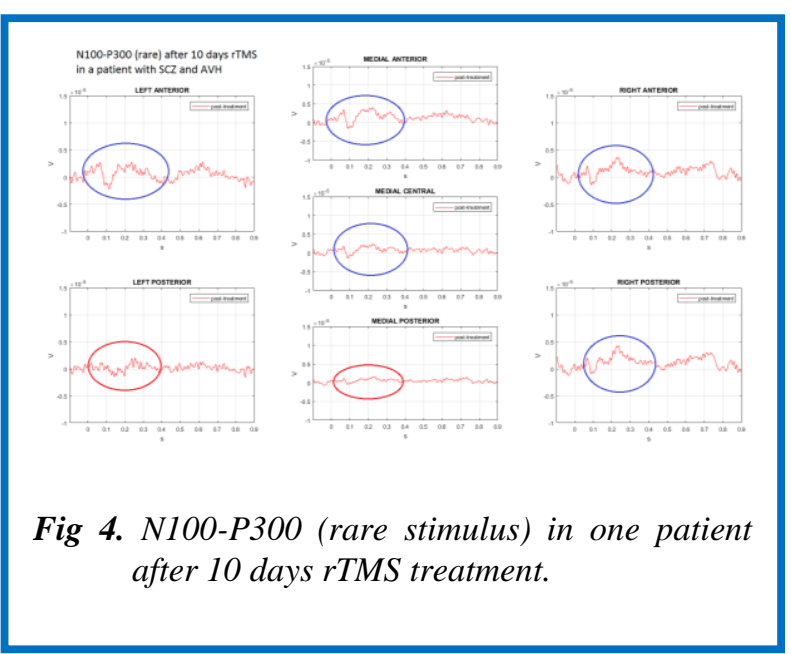


treatment (Figure 4). Even so, the automatic maximum minimum voltage measurements for 15 electrodes in each ROI detected higher responses in the patients group in few occasions which at visual inspection resulted to be erroneous due to original signal difficult acquisition or data processing. P50 suppression ratio analyzed in one patient after 10 days rTMS treatment at left temporoparietal region (T3-P3 EEG location) was increased, which is in contradiction to our hypothesis.

The main limitations of the present study are the limited sample size, the lack of a comparator group, the patients' symptoms and medications, which are not presented in this work and the data processing, mostly of P50 waves which are of order of microvolts. It would be of interest to assess N100 gating responses from the same datasets as for P50 due to better visibility and higher amplitude. P300 deviant stimulus absent responses in patients group in baseline condition might be related to the fact that we did not performed classical P300 oddball task with requirement of motor movements or counting of rare stimuli in order to not alter the cortical responses in motor areas or parietal lobes. P300 waves obtained in healthy participants and in two patients after rTMS treatment might be represented by P300a (220-280 ms) related to a novelty or context updating paradigm.

In conclusion, we proved that recording P50 and ERP is possible using HD-EEG 256 channels and the results in healthy subjects are reproducible and show similar topography when studying the different regions of interests. In the patients group we observed that P50 waves are very difficult to measure and the relatively small amplitude or the gated responses cannot be measured with our protocol. A lot of data will have to be excluded from the final analysis. N100-P300 complex is measurable for all selected regions and it appears to be a helpful neurophysiological marker in assessing if ERPs components change after rTMS treatment at the stimulation site or in other different cortical areas.

\section{List of acronyms}

AVH - Auditory verbal hallucinations

ERP - Event-related potentials

ROI - Region of interest

rTMS - Repetitive transcranial magnetic stimulation

\section{Authors contributions}

S.M., E.P., E.Í., R.A. and O.C.B. processed the experimental data and performed the analysis, S.M., V.D.J., A.D.J., E.Í., E.P., R.A. and O.C.B. performed the measurements, A.D.J, E.Í and O.C.B. performed the 10 days TMS treatments, P.G. O.C.B. and V.D.J. were involved in planning and supervised the work, O.C.B., S.M., E.P. and R.A. drafted the manuscript and designed the figures. All authors discussed the results and commented on the manuscript.

\section{Acknowledgments}

The authors wish to thank the University Hospital Landspitali in Reykjavík for infrastructural support.

\section{Funding}

None.

\section{Conflict of Interest}

The authors declare they have no conflicts of interest.

\section{Ethical Publication Statement}

We confirm that we have read the Journal's position on issues involved in ethical publication and affirm that this report is consistent with those guidelines.

\section{Corresponding Author}

Ovidiu C. Banea, MD, National University Hospital of

Iceland, 108 Fossvogur, Reykjavík, Iceland

ORCID iD: 0000-0002-0074-7011

Email: ovidiubanea@gmail.com,ovidiuc@landspitali.is

Email and ORCID iD of co-authors

Sara Marcu: saramarcu95@gmail.com

ORCID iD: 0000-0001-7975-0741

Elena Pegolo: elena.pegolo@gmail.com

ORCID iD: 0000-0003-2443-3663

Eysteinn Ívarsson: eysteinn.ivarsson@gmail.com

ORCID iD. 0000-0001-9527-4933

Aron D. Jónasson: arondalin@gmail.com

ORCID iD: 0000-0002-1414-0157

Viktor D. Jónasson: viktordiar91@gmail.com

ORCID iD: 0000-0002-5756-4865

Romain Aubonnet: aubo.rom@gmail.com

ORCID iD: 0000-0002-5395-775X

Paolo Gargiulo: paologar@landspitali.is

ORCID iD: 0000-0002-5049-4817

\section{References}

1. Bramon E, Rabe-Hesketh S, Sham P, et al. Metaanalysis of the P300 and P50 waveforms in schizophrenia. Schizophr Res 2004;70:315-29. doi: 10.1016/j.schres.2004.01.004.

2. Thoma L, Rentzsch J, Gaudlitz K, et al. P50, N100, and P200 Sensory Gating in Panic Disorder. Clin EEG Neurosci 2020;51: doi:10.1177/15500 59419899324.

3. Adler LE, Pachtman E, Franks RD, et al. Neurophysiological evidence for a defect in neuronal mechanisms involved in sensory gating in schizophrenia. Biol Psychiat 1982;17:639-54. doi: 10.1016/0006-3223(85)90113-1.

4. Sánchez-Morla EM, Santos JL, Aparicio A, et al. Neuropsychological correlates of P50 sensory gating in patients with schizophrenia. Schizophr Res 2013;143:102-6. doi: 10.1016/j.schres.2012. 10.017 .

5. Potter D, Summerfelt A, Gold J, Buchanan RW. Review of Clinical Correlates of P50 Sensory Gating Abnormalities in Patients with 


\section{High density EEG to assess TMS treatment in schizophrenia}

Eur J Transl Myol 30 (1): 134-138, 2020

Schizophrenia. Schizophr Bull 2006; 32:692-700. doi: 10.1093/schbul/sbj050.

6. Ogura C, Nageishi Y, Matsubayashi M, et al. Abnormalities in Event-Related Potentials, N100, P200, P300 and Slow Wave in Schizophrenia. Psychiat Clin Neuros 1991;45:57-65. doi: 10.1111 /j.1440-1819.1991.tb00506.x.

7. Earls HA, Curran T, Mittal V. A Meta-analytic Review of Auditory Event-Related Potential Components as Endophenotypes for Schizophrenia: Perspectives From First-Degree Relatives. Schizophr Bull 2016;42:1504-16. doi: 10.1093 /schbul/sbw047.

8. Hoffman RE, Boutros NN, Berman RM, et al. Transcranial magnetic stimulation of left temporoparietal cortex in three patients reporting hallucinated "voices." Biol Psychiat 1999;46: 130 2. doi: 10.1016/s0006-3223(98)00358-8.

9. Sommer IEC, Slotema CW, Daskalakis ZJ, et al. The treatment of hallucinations in schizophrenia spectrum disorders. Schizophr Bull 2012;38: 70414. doi: $10.1093 / \mathrm{schbul} / \mathrm{sbs} 034$.

10. Slotema CW, Blom JD, Sommer IEC. [Treatment strategies for auditory verbal hallucinations]. Tijdschr Psychiatr 2014;56: 247-56.

11. Lefaucheur J-P, André-Obadia N, Antal A et al. Evidence-based guidelines on the therapeutic use of repetitive transcranial magnetic stimulation (rTMS). Clin Neurophysiol 2014;125:2150-2206. doi: 10.1016/j.clinph.2014.05.021.

12. Morstyn R, Duffy FH, McCarley RW. Altered P300 topography in schizophrenia. Arch Gen Psychiat 1983;40:729-34. doi: 10.1001/archpsyc .1983.01790060027003.

13. Salisbury DF, Shenton ME, McCarley RW. P300 topography differs in schizophrenia and manic psychosis. Biol Psychiat 1999;45:98-106. doi: 10.1016/s0006-3223(98)00208-x.

14. Kurthen M, Trautner P, Rosburg T et al. Towards a functional topography of sensory gating areas: invasive P50 recording and electrical stimulation mapping in epilepsy surgery candidates. Psychiat Res 2007;155:121-33. doi: 10.1016/j.pscychresns .2006.10.003.
15. Light GA, Williams LE, Minow $F$, et al. Electroencephalography (EEG) and Event-Related Potentials (ERP's) with Human Participants. Curr Protoc Neurosci 2010;52:1-6. doi: 10.1002/0471 142301.ns0625s52.

16. Hall M-H, Taylor G, Salisbury DF, Levy DL. Sensory gating event-related potentials and oscillations in schizophrenia patients and their unaffected relatives. Schizophr Bull. 2011;37:1187-99. doi: 10.1093/schbul/sbq027.

17. Olincy A, Braff DL, Adler LE, et al. Inhibition of the P50 cerebral evoked response to repeated auditory stimuli: results from the Consortium on Genetics of Schizophrenia. Schizophr Res 2010;119:175-82. doi: 10.1016/j.schres.2010.03. 004.

18. Stefánsson SB, Jónsdóttir TJ. Auditory eventrelated potentials, auditory digit span, and clinical symptoms in chronic schizophrenic men on neuroleptic medication. Biol Psychiat 1996;40:1927. doi: 10.1016/0006-3223(95)00351-7

19. Tadel F, Baillet S, Mosher JC, Pantazis D, Leahy RM. Brainstorm: a user-friendly application for MEG/EEG analysis. Comput Intell Neurosci. 2011;2011: 879716. doi: 10.1155/2011/879716.

20. Ívarsson E, Shaw A, Georgsdóttir AÓ et al. A Novel Technique to Trigger High Beta and Low Gamma Activity in Patients with Schizophrenia. IFMBE Proceedings. 2020. pp. 1064-70. doi:10. 1007/9783-030-31635-8_129

21. Banea OC, Pegolo E, Marcu S et al. P50 and P300 Event Related Potentials in Patients with Schizophrenia Recorded from High-Density EEG. IFMBE Proceedings. 2020. pp. 1071-77. doi:10. 1007/978-3-030-31635-8_130

22. Edmunds KJ, Petersen H, Hassan M et al. Cortical recruitment and functional dynamics in postural control adaptation and habituation during vibratory proprioceptive stimulation. J Neural Eng 2019;16:026037. doi: 10.1088/1741-2552/ab0678.

Submitted: February 15, 2020

Revision received: March 4, 2020 Accepted for publication: March 4, 2020 